\title{
Prostate Segmentation with Texture Enhanced Active Appearance Model
}

\author{
Soumya Ghose ${ }^{* \dagger}$, Arnau Oliver ${ }^{\dagger}$, Robert Martí ${ }^{\dagger}$, Xavier Lladó ${ }^{\dagger}$, Jordi Freixenet ${ }^{\dagger}$, Joan C.Vilanova ${ }^{\ddagger}$ and \\ Fabrice Meriaudeau* \\ *Laboratoire Le2I - UMR CNRS 5158, Université de Bourgogne, 12 Rue de la Fonderie, 71200 Le Creusot, France. \\ E-mail: soumyaghose@gmail.com, fabrice.meriaudeau@u-bourgogne.fr. \\ ${ }^{\dagger}$ Computer Vision and Robotics Group, University of Girona, Campus Montilivi, Edifici P-IV, \\ Av. Lluís Santaló, s/n, 17071 Girona, Spain. \\ E-mail: aoliver@eia.udg.edu, marly@eia.udg.edu, llado@eia.udg.edu,jordif@eia.udg.edu. \\ ${ }_{\ddagger}^{\ddagger}$ Clinica Girona, Calle Joan Maragall 26, 17002 Girona, Spain. \\ E-mail: kvilanova@comg.es.
}

\begin{abstract}
Prostate contour segmented from Trans Rectal Ultra Sound (TRUS) and Magnetic Resonance (MR) images could improve inter-modality registration accuracy and reduce computational complexity of the procedure. However, prostate segmentation in each of these modalities is a challenging task in presence of imaging artifacts, intensity heterogeneities, and large inter patient shape variabilities of the prostate. We propose to use Haar wavelet approximation coefficients to extract texture features of the prostate region in both modalities to guide a deformable parametric model to segment the prostate in a multi-resolution framework. Principal Component Analysis (PCA) of the shape and texture information of the prostate region obtained from the training data aids contour propagation of the deformable parametric model. Prior knowledge of the optimization space is utilized for optimal segmentation of the prostate. Our method achieves a mean Dice Similarity Coefficient (DSC) value of $0.95 \pm 0.01$, with mean segmentation time of $0.72 \pm 0.05$ seconds in a leave-one-out validation framework with 25 TRUS images grabbed from a video sequence. DSC value of $0.88 \pm 0.06$ with a mean segmentation time of $0.81 \pm 0.02$ seconds was recorded for MR images when validated with 15 central slice images of 15 datasets from the MICCAI prostate segmentation challenge 2009. Our proposed method performs computationally efficient accurate multi-modal prostate segmentation in presence of intensity heterogeneities and imaging artifacts.
\end{abstract}

\section{INTRODUCTION}

Index Terms-Prostate biopsy, TRUS/MR registration, Haar wavelets, Active Appearance Model.

\section{INTRODUCTION}

Prostate cancer is the most commonly diagnosed cancer in North America accounting for over 33,000 deaths every year [1]. In clinical practice TRUS guided needle biopsy is performed to confirm the presence of cancerous tissues in prostate. However, localization of malignant tissues in TRUS images are difficult due to low Signal to Noise Ratio (SNR). Tissue samples are collected from different zones of the prostate to maximize the chance of locating malignant tissues. Superior contrast of soft tissues of the prostate gland in MR images facilitate improved detection of cancerous tissues. However, interventional MRI guided biopsy is expensive and complicated. Hence the solution lies in the fusion of the two imaging modalities to exploit the high quality of MR images in TRUS interventional biopsies.

Accurate segmentation of the prostate in TRUS and MR images could improve registration accuracy and reduce computational complexities involved with the procedure [2]. However, segmentation of the prostate in each of these modalities have challenges associated with them. Low SNR in TRUS images produces broken and discontinuous prostate edges with traditional edge detection filters like Sobel, Prewitt and Canny. Intensity heterogeneities inside the prostate gland inhibits the design of a global descriptor for the prostate based on intensity. Added to these, presence of speckle noise, shadow artifacts, and micro-calcification significantly affects the segmentation accuracy of the prostate in TRUS images. MR images produce superior contrast of the soft tissues of the prostate gland compared to TRUS images. However detail information of the prostate gland introduces large inter patient shape and intensity variabilities. Presence of heterogeneous intensity distribution inside the prostate gland produces broken and false edges with edge detection filters and inhibits the design of a global descriptor for the prostate region. Large inter patient shape variabilities of the prostate in MR images reduces segmentation accuracy of algorithms designed on shape prior basis. Hence, it is useful to incorporate both shape and intensity priors in segmentation algorithms to improve accuracy in both modalities.

Cootes et al. [3] proposed an efficient framework to combine shape and intensity prior in their Active Appearance Model(AAM). Medina et al. [4] used AAM to segment prostate in two Dimensional (2D) TRUS images with an overlap ratio of $96 \%$. Since then, not many researchers have used AAM for prostate segmentation in TRUS and MR images. It is argued by Wolstenholme and Taylor [5] that the time complexity involved with AAM is high and is unsuitable for real time procedures. Instead, they proposed to use wavelet coefficients of the training images to build the AAM. Larsen et al. [6] showed that frequency separation in wavelet transform allowed an edge enhancement that provided better result in terms of segmentation accuracy compared to traditional 
AAMs. They proposed to use a texture vector comprising the truncated detail and approximation coefficients in a multiresolution framework for AAM propagation.

To address the challenges involved with prostate segmentation in MR and TRUS images, we propose a novel AAM that is propagated by the approximation coefficients of Haar wavelet transform. The approach is similar to that of Larsen et al. [6] applied to face segmentation. However, deviating from their model of using approximation and truncated detail coefficient of Haar wavelet for AAM propagation, we propose to discard the detail coefficients and use approximation coefficients for AAM propagation to reduce computational complexity. The performance of our method is validated with 25 TRUS images grabbed from TRUS video sequence and with $15 \mathrm{MR}$ datasets of MICCAI prostate segmentation challenge 2009. Experimental result reveals that the method is robust and delivers good results in presence of low SNR, intensity heterogeneities, presence of micro-calcification and large shape variabilities.

The rest of the paper is organized as follows. The texture driven AAM is formulated in Section II followed by quantitative and qualitative evaluation of our method in Section III. We finally draw conclusions in Section IV.

\section{Methods}

The proposed method is built on two major components: the adaptation of AAM to incorporate texture features and the generation of texture features using the Haar wavelet transform. We present the traditional AAM first, followed by a comprehensive discussion about using Haar wavelet to extract texture features for the prostate region.

\section{A. Active Appearance Model}

AAM provides a compact parametric framework utilizing prior shape and intensity variabilities learned from a training model to segment an unseen test image exploiting the prior knowledge of the nature of the optimization space [6]. The shape model built from manually segmented contours and the appearance model built from intensity distributions inside the manually segmented regions are combined to produce traditional AAM that incorporates prior knowledge of shape and intensity variabilities in the segmentation framework for the object under study.

The creation of the shape model is initiated with the formation of the Point Distribution Model (PDM) [7] built from manually segmented contours. Generalized Procrustes Analysis (GPA) of the PDMs is used to minimize the differences between the PDMs and align the PDMs to a common reference frame. PCA of the aligned PDMs is used to identify the principal components of the variations in shape and suppress redundancy. Intensity distributions are warped into correspondence using a piece wise affine warp and sampled from shape free reference. PCA of the intensity distribution is used to identify the principal components of intensity variations.

The shape model may be formalized in the following manner. Let each shape be represented by a vector $x$ with $n$ points of the PDM as given in (1).

$$
\begin{aligned}
x & =\left[x_{1}, x_{2}, \ldots, x_{n} ; y_{1}, y_{2}, \ldots, y_{n}\right] \\
x_{s} & =\bar{x}+\Phi_{s} b_{s}
\end{aligned}
$$

where the shape model $x_{s}$ is a function of the mean model $\bar{x}$, the matrix $\Phi_{s}$ includes the eigenvectors and a set of shape deformation parameters are present in $b_{s}$.

The appearance model is formalized as follows. In (2) the normalized pixel intensities are given by a vector $g$ of $m$ samples obtained using a piece-wise affine warp based on Delaunay triangulation of the mean shape. Normalization of the texture information is performed followed by PCA of the intensities to generate the appearance model $g_{i}$, where $\bar{g}$ represents the mean intensity model, a matrix $\Phi_{g}$ that includes the eigenvectors of intensity variations and a set of parameters $b_{g}$ for intensity variations.

$$
\begin{aligned}
g & =\left[g_{1}, g_{2}, \ldots, g_{m}\right] \\
g_{i} & =\bar{g}+\Phi_{g} b_{g}
\end{aligned}
$$

Correlation between the shape and intensity variations is used to produce a concatenated vector of shape and intensity variations. In (3), $b$ represents the concatenated vector where $W_{s}$ is a diagonal matrix whose entries are the weight parameters that account for the differences of units between the shape and pixel intensities. A third PCA is applied to this vector to produce the combined model $b_{f}$ where $Q$ is a matrix of eigenvectors and $c$ is a vector of appearance model parameters controlling both shape and intensity variations of the model.

$$
\begin{aligned}
b & =\left(\begin{array}{c}
W_{s} b s \\
b_{g}
\end{array}\right)=\left(\begin{array}{c}
W_{s} \phi_{s}^{T}(x-\bar{x}) \\
\phi_{s}^{T}(g-\bar{g})
\end{array}\right) \\
b_{f} & =Q c
\end{aligned}
$$

Shapes and intensities are then synthesized from (3) as shown in (4)

$$
\begin{aligned}
x & =\bar{x}+\Phi_{s} W_{s}^{-1} Q_{s} c \\
g & =\bar{g}+\Phi_{g} Q_{g} c
\end{aligned}
$$

The AAM is optimized minimizing the difference between the input and the synthesized images [3]. This problem is computationally expensive. Hence, to reduce the computational time we propose to use the approximation coefficients of Haar wavelet transform in place of the raw intensities. This will introduce the additional time requirement of transformation of the image into a new representation. However, as stated in [6] the computational burden can be considerably reduced if the transformation is based on sparse matrix.

\section{B. Haar Wavelets for Texture Analysis}

Wavelets are a family of basis functions that decomposes signal into frequency and time domains in a multi-resolution framework. A set of linear, rank preserving convolution operations are carried out to decompose an image by a low pass and high pass filters. Three detail coefficient sub-bands 
corresponding to horizontal, vertical and diagonal edges are generated by the high pass filters in a $2 \mathrm{D}$ image. The low pass filter generates the approximation coefficients which is downsampled and is further decomposed to analyze the detail and the approximation coefficients at a coarser resolution.

To introduce wavelet coefficients in AAM, we formalize the framework with the used notation. First, let a n-level wavelet transform be denoted by

$$
W(t)=\kappa t=\widehat{w}=\left[\widehat{a}^{T}, \widehat{u}_{1}^{T}, \ldots, \widehat{u}_{n}^{T}\right]^{T}
$$

where $\kappa$ is the Haar wavelet filter, $\widehat{a}$ and $\widehat{u}$ represent the approximation and the detail coefficients respectively, and $\widehat{w}$ is the wavelet transformed image [6]. The detail coefficients are suppressed to produce a truncated wavelet basis as

$$
b(\widehat{w})=C \widehat{w}=\left[\widehat{a}^{T}, 0, \ldots 0,,\right]^{T}
$$

where $C$ corresponds to a modified identity matrix with its rows corresponding to the detail coefficients removed. The AAM is built on the truncated wavelet basis constituting the texture. The PCA of the texture is given by

$$
a=\bar{a}+\Phi_{a} b_{w}
$$

where, $\bar{a}$ is the mean of the approximation coefficients, $\Phi_{a}$ is the matrix of eigenvectors and $b_{w}$ is the matrix of corresponding to set of parameters for the approximation coefficients variations.

Suppression of the high frequency components reduces texture information. However, texture information that has to be preserved is context dependent. Suppression of the detail coefficients reduces speckle noise and micro-calcifications and produces an uniform texture map of the prostate. Suppressing the detail coefficients reduces high gradient components inside the prostate gland reducing inter patient variabilities in TRUS and MR images. Finally, such suppression reduces the computational complexities involved with the fitting of a new image to the model. It is to be noted that, the approximation coefficients preserve significant texture information [8] and PCA of the approximation coefficients facilitates suppresion of noise in the underlying texture with the preservation of important components.

As stated by Larsen et al. [6] the multi-level decomposition of the image in wavelet domain propagates the fitting error due to loss of texture information. Hence, we have adopted wavelet decomposition of the first level and subsequently fitted our model to the approximation coefficients in coarser to finer spatial resolutions to reduce the texture dependent fitting error.

The mean model is initialized by clicking in any position close to the center of the prostate decided on visual inspection. The mean model initialization and subsequent multi-resolution segmentations are produced based on the approximation coefficients of the Haar wavelet.
TABLE I

Prostate Segmentation Evaluation Metreics for TRUS and MR IMAGES

\begin{tabular}{|l|l|l|l|l|l|}
\hline & DSC & MAD $(\mathrm{mm})$ & Specificity & Sensitivity & Time(secs) \\
\hline TRUS & $0.95 \pm 0.01$ & $1.48 \pm 0.36$ & $0.92 \pm 0.02$ & $0.998 \pm 0.001$ & $0.72 \pm 0.05$ \\
\hline MRI & $0.88 \pm 0.06$ & $3.97 \pm 2.74$ & $0.86 \pm 0.11$ & $0.998 \pm 0.001$ & $0.81 \pm 0.02$ \\
\hline
\end{tabular}

TABLE II

Comparison of Our Method With Traditional AAM

\begin{tabular}{|c|c|c|c|c|}
\hline & \multicolumn{2}{|c|}{ TRUS } & \multicolumn{2}{c|}{ MRI } \\
\cline { 2 - 5 } & Our Method & Traditional AAM & Our Method & Traditional AAM \\
\hline DSC & $0.95 \pm 0.01$ & $0.95 \pm 0.02$ & $0.88 \pm 0.06$ & $0.88 \pm 0.08$ \\
\hline MAD & $1.48 \pm 0.36$ & $1.54 \pm 0.74$ & $3.97 \pm 2.74$ & $4.05 \pm 2.74$ \\
\hline HD & $5.08 \pm 1.19$ & $5.27 \pm 2.83$ & $9.56 \pm 4.5$ & $9.68 \pm 4.2$ \\
\hline Specificity & $0.92 \pm 0.02$ & $0.91 \pm 0.04$ & $0.86 \pm 0.11$ & $0.84 \pm 0.12$ \\
\hline Sensitivity & $0.998 \pm 0.001$ & $0.998 \pm 0.001$ & $0.998 \pm 0.001$ & $0.995 \pm 0.005$ \\
\hline Time & $0.72 \pm 0.05$ & $0.87 \pm 0.02$ & $0.81 \pm 0.02$ & $0.77 \pm 0.02$ \\
\hline
\end{tabular}

\section{EXPERIMENTAL RESULTS}

We have validated the accuracy and robustness of our approach on a series of 25 prostate ultrasound images and $15 \mathrm{MR}$ images using a leave-one-out evaluation strategy. The TRUS images are of resolution $538 \times 418$ pixels and are grabbed from TRUS video sequences (acquired with a Siemens Aquson). The 15 central slice MR images of 15 datasets are from the MICCAI prostate segmentation challenge 2009 [9] with a resolution of $256 \times 256$ pixels. Our method was implemented in Matlab 7 on a Intel Core 2 Duo T5250 processor of 1.5 Ghz processor speed and 2 GB RAM. We have used most of the popular prostate segmentation evaluation metrics such as the Dice similarity coefficient (DSC), Mean Absolute Distance (MAD), specificity and sensitivity in order to evaluate our approach. The average values for all the 25 TRUS images and $15 \mathrm{MR}$ images along with average segmentation time (Time) in seconds are given in Table I.

We have used the evaluation metrics of Table I and 95\% Hausdorff Distance (HD) to compare our method against traditional AAM. The experimental setup, manner of execution and the TRUS and MR datasets are identical. The evaluation results are given in Table II. It is observed from Table II that DSC values for both the methods are comparable. However, our method performs better when compared in terms of MAD, HD and specificity. The variances observed in different metrics are significantly higher in traditional AAM justifying the repeatability of our method. The computational complexities involved with our method is marginally higher than the traditional AAM. This is due to the reason that traditional AAM works with raw intensities and in our method, a transformation of the test image into wavelet domain is necessary before fitting the AAM.

Qualitative evaluation is done to demonstrate the robustness of the proposed method against low SNR, intensity heterogeneities, shadow artifacts, speckle noise and microcalcification in TRUS images and inter patient shape variabilities for MR images. In all the images the green contours depict the ground truth and red contours show the obtained result. As observed in Fig. 1(b), during the initialization a section of the mean model (blue contour) is located in a region of 


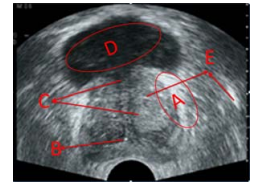

(a)

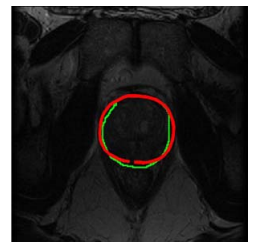

(d)

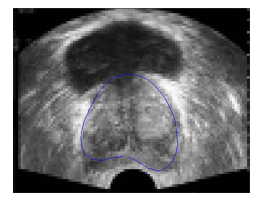

(b)

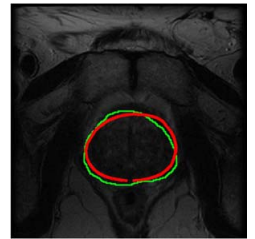

(e)

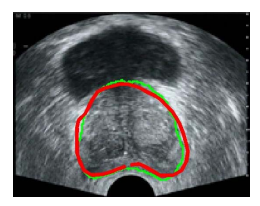

(c)

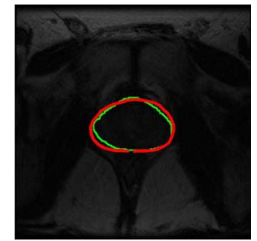

(f)
Fig. 1. Qualitative evaluation of segmentation. (a) Prostate artifacts in TRUS image of the prostate, $\mathrm{A}=$ Low $\mathrm{SNR}, \mathrm{B}=$ Micro-Calcification, $\mathrm{C}=$ Intensity difference inside prostate region, $\mathrm{D}=$ Shadow Artifacts, $\mathrm{E}=$ Speckle Noise. (b) Manual initialization of the mean model (blue contour) by clicking on prostate center, (c) Final segmentation result. Manual segmentation shown with green contour and the red contour show the segmentation achieved, (d), (e) and (f) show some of the results in MRI.

shadow artifact in the TRUS image, the model successfully avoids the artifacts and segments the prostate accurately with a DSC value of 0.94 . The robustness of the model against large prostate shape variabilities and intensity heterogeneities inside the prostate region in MR images is demonstrated in Fig. 1(d), 1(e), 1(f). Our method successfully segments the prostate with reasonable DSC values of $0.93,0.89$ and 0.95 respectively.

The mean model intializations in all the TRUS and MR images are done on visual inspections and therefore varies from one case to the other. However, low standard deviation values of DSC, specificity and sensitivity being $0.01,0.015$ and 0.0001 respectively for TRUS images and being $0.06,0.11$ and 0.0001 respectively for MR images, indicate that the accuracy of the final segmentation results using our method is indifferent to the manual initialization of the mean model. The mean model could be initialized automatically on the assumption that the prostate is visible in the center of the TRUS and MR images. A more sophisticated approach would be an initial rough classification of the TRUS and MR images to identify the prostate regions and then initialize the mean model at the center of the prostate.

Comparison of different prostate segmentation methodologies is difficult in absence of public datasets and standardized evaluation metrics. In addition, the methods are developed with a wide variety of algorithms with specific application requirements. Nevertheless, to have an overall qualitative estimate of the functioning of our method we have compared our method with some of the state-of-the-art works in literature in Table III for TRUS and MR images. Please note '-' in Table III means information is not available.

For TRUS images we observe that our mean segmentation time of $0.72 \pm 0.05$ seconds is comparable to some of the works in literature and computationally expensive only compared to Yan et al. [2]. Note that our method is implemented in Matlab and Yan et al. code is optimized using dynamic programming in a $\mathrm{C}++$ and ITK framework. We achieved an average DSC value of $0.95 \pm 0.01$ that is less than the area overlap measure of Abolmaesumi et al. [1] of $98 \%$. However, we have used more images (25 images) compared to 6 images of Abolmaesumi et al. Our MAD value of $1.48 \pm 0.36 \mathrm{~mm}$ is very close to Gong et al. [10] average distance value of $1.36 \pm 0.6 \mathrm{~mm}$.

For MR images our segmentation time is comparable to the methods in Table III. Our average DSC value is comparable to Klein et al. [15] but less than Samiee et al. [14]. However, it is to be noted that we have used more datasets (15 datasets) compared to Samiee et al. (2 datasets). The performance of AAM improves with the use of large shape and intensity variabilities during training. However, with a small dataset of 15 images for building the model in a leave-one-out approach, our method performs well considering the large variabilities present in the prostate shapes. Comparing our results with some of the works in literature, we can state that our method performs accurate and computationally efficient prostate segmentation.

\section{Conclusions}

A novel approach of AAM propagation using Haar wavelet approximation coefficients with the goal of segmenting the prostate in 2D TRUS and MR images has been proposed. Our approach is accurate, computationally efficient and robust to low SNR, intensity heterogeneities of prostate tissues, shadow artifacts, speckle noise and micro-calcifications in TRUS images and large inter patient prostate shape variabilities in MR images. While the proposed method is validated with prostate mid-gland images, the effectiveness of the method against base and apical region slices is yet to be validated. We would like to explore the possibilites of using 3D AAM for prostate segmentation in MR volume. Computational time of our process is fast but not yet suitable for real-time applications like MRI-TRUS fusion. We would like to explore the possibilities of optimizing our code and using the CUDA platform that would achieve necessary hardware acceleration and parallelization in order to produce real-time 2D fusion of TRUS and MR images of the prostate.

\section{ACKNOWLEDGMENT}

This research is a part of the PROSCAN project of the VICOROB laboratory of University of Girona, Catalunya, Spain. The authors would like to thank VALTEC 08-1-0039 of Generalitat de Catalunya, Spain and Conseil Regional de Bourgogne, France for funding this research.

\section{REFERENCES}

[1] P. Abolmaesumi and M. Sirouspour, "Segmentation of Prostate Contours from Ultrasound Images," IEEE International Conference on Acoustics, Speech, and Signal Processing, vol. 3, pp. 517-520, 2004.

[2] P. Yan, S. Xu, B. Turkbey, and J. Kruecker, "Optimal Search Guided by Partial Active Shape Model for Prostate Segmentation in TRUS Images," Proceedings of the SPIE Medical Imaging : Visualization, Image-Guided Procedures, and Modeling, vol. 7261, pp. 72611G-72611G-11, 2009.

[3] T. Cootes, G. Edwards, and C. Taylor, "Active Appearance Models," Book Series Lecture Notes in Computer Science Springer, vol. 1407, pp. 484-498, 1998. 
TABLE III

Prostate Segmentation Evaluation Metrics Comparison For TRUS and MR IMAGes

\begin{tabular}{|c|c|c|c|c|c|c|}
\hline & Reference & Year & Area Overlap Accuracy & Contour Accuracy & Time & Datasets \\
\hline$\underset{⿱ ⺌}{\mathscr{2}}$ & $\begin{array}{c}\text { Yan[2] } \\
\text { Abolmaesumi[1] } \\
\text { Gong[10] } \\
\text { Betrouni[11] } \\
\text { Shen[12] } \\
\text { Ladak[13] } \\
\text { Our Method }\end{array}$ & $\begin{array}{l}2009 \\
2004 \\
2004 \\
2004 \\
2003 \\
2000\end{array}$ & $\begin{array}{c}\text { Average Area overlap } 98 \% \\
- \\
\text { Average Area overlap } 93 \pm 0.9 \% \\
\text { Average Area overlap error } 3.98 \pm 0.97 \% \\
\text { Average Area accuracy } 90.1 \pm 3.2 \% \\
\text { Average DSC } 0.95 \pm 0.01\end{array}$ & $\begin{array}{c}\text { Average MAD } 1.79 \pm 0.95 \mathrm{~mm} \\
- \\
\text { Mean error } 1.36 \pm 0.6 \mathrm{~mm} \\
\text { Average distance } 3.77 \pm 1.3 \text { pixels } \\
\text { Average distance } 3.2 \pm 0.87 \text { pixels } \\
\text { Average MAD } 4.4 \pm 1.8 \text { pixels } \\
\text { Average MAD } 1.48 \pm 0.36 \mathrm{~mm}\end{array}$ & $\begin{array}{c}0.3 \text { secs } \\
\text { less than } 1 \mathrm{sec} \\
\text { less than } 5 \text { secs } \\
\text { less than } 5 \text { secs } \\
- \\
- \\
0.72 \pm 0.05 \text { secs }\end{array}$ & $\begin{array}{l}10 \text { datasets } \\
6 \text { images } \\
125 \text { images } \\
10 \text { images } \\
8 \text { images } \\
117 \text { images } \\
25 \text { images }\end{array}$ \\
\hline 를 & $\begin{array}{c}\text { Samiee[14] } \\
\text { Klein[15] } \\
\text { Our Method }\end{array}$ & $\begin{array}{l}2006 \\
2008\end{array}$ & $\begin{array}{c}\text { Average DSC } 0.9057 \pm 0.0014 \\
\text { Median DSC } 0.85 \\
\text { Average DSC } 0.88 \pm 0.06\end{array}$ & $\begin{array}{c}- \\
- \\
\text { Average MAD } 3.97 \pm 2.74 \mathrm{~mm}\end{array}$ & $\begin{array}{c}5 \text { secs } \\
15 \text { minutes } \\
0.81 \pm 0.02 \text { secs }\end{array}$ & $\begin{array}{l}2 \text { datasets } \\
50 \text { datasets } \\
15 \text { datasets }\end{array}$ \\
\hline
\end{tabular}

[4] R. Medina., A. Bravo, P. Windyga, J. Toro, P. Yan, and G. Onik, "A 2D Active Appearance Model For Prostate Segmentation in Ultrasound Images," 27th Annual International Conference of the IEEE Engineering in Medicine and Biology Society, pp. 3363-3366, 2005.

[5] C. B. H. Wolstenholme and C. J. Taylor, "Wavelet Compression of Active Appearance Models," Book Series Lecture Notes in Computer Science Springer, vol. 1679, pp. 544-554, 1999.

[6] R. Larsen, M. B. Stegmann, S. Darkner, S. Forchhammer, T. F. Cootes, and B. K. Ersbll, "Texture Enhanced Appearance Models," Computer Vision and Image Understanding, vol. 106, pp. 20-30, 2007.

[7] T. F. Cootes, A. Hill, C. J. Taylor, and J. Haslam, "The Use of Active Shape Model for Locating Structures in Medical Images," Image and Vision Computing, vol. 12, pp. 355-366, 1994.

[8] M. Petrou and P. G. Sevilla, Image Processing: Dealing With Texture, 1st ed. Wiley, 2006.

[9] MICCAI, "2009 prostate segmentation challenge MICCAI," http://wiki.na-mic.org/Wiki/index.php, 2009.

[10] L. Gong, S. D. Pathak, D. R. Haynor, P. S. Cho, and Y. Kim, "Parametric Shape Modeling Using Deformable Superellipses for Prostate Segmentation," IEEE Transactions on Medical Imaging, vol. 23, pp. 340-349, 2004.

[11] N. Betrouni, M. Vermandel, D. Pasquier, S. Maouche, and J. Rousseau, "Segmentation of Abdominal Ultrasound Images of the Prostate Using A priori Information and an Adapted Noise Filter," Computerized Medical Imaging and Graphics, vol. 29, pp. 43-51, 2005.

[12] D. Shen, Y. Zhan, and C. Davatzikos, "Segmentation of Prostate Boundaries from Ultrasound Images Using Statistical Shape Model," IEEE Transactions on Medical Imaging, vol. 22, pp. 539-551, 2003.

[13] H. M. Ladak, F. Mao, Y. Wang, D. B. Downey, D. A. Steinman, and A. Fenster, "Prostate Segmentation from 2D Ultrasound Images," Proceedings of the 22nd Annual International Conference of the IEEE Engineering in Medicine and Biology Society, vol. 4, pp. 3188-3191, 2000.

[14] M. Samiee, G. Thomas, and R. Fazel-Rezai, "Semi-Automatic Prostate Segmentation of MR Images Based on Flow Orientation," IEEE International Symposium on Signal Processing and Information Technology, pp. 203-207, 2006.

[15] S. Klein, U. A. van der Heide, I. M. Lipps, M. V. Vulpen, M. Staring, and J. P. W. Pluim, "Automatic Segmentation of the Prostate in 3D MR Images by Atlas Matching Using Localized Mutual Information," Medical Physics, vol. 35, pp. 1407-1417, 2008. 